\title{
A Web-Based Mashup Tool for Information Integration and Delivery to Mobile Devices
}

\author{
Prach Chaisatien and Takehiro Tokuda \\ Department of Computer Science, Tokyo Institute of Technology \\ Meguro, Tokyo 152-8552, Japan \\ \{prach, tokuda\}@tt.cs.titech.ac.jp
}

\begin{abstract}
The immense popularity in mobile internet has driven part of classic web information and services to transform and shrink themselves to match the diversity of web-capable mobile devices. This paper will present a Web-based mashup tool to work with range of Japanese domestic mobile handsets and their variety of limited functionalities. Our purpose is to provide users an alternative option to customize information to be displayed and to integrate available services for their mobile phone. Three major working components, functionality tester, service aggregator and output simulator will aid users in understanding the capability of their mobile device and selecting corresponding functionalities. Successfully simulated and simplified information services will be accessible via user's mobile phones with correct configurations.
\end{abstract}

Keywords: Mobile Devices, Web-based, Customization, Integration, Mashup.

\section{Introduction}

The popularity of mobile phones has begun to build up due to their capability of web access. One of the great challenges for mobile developers is dealing with devices' specification. A web page of application which works and displays correctly on one mobile phone may not work or display correctly on another. Classic web information and service may not be easily accessible on a mobile phone. In this paper, we are proposing a web-based tool which can help users build a mashup mobile application from specific information sources, select integratible web services and compile them into his/her personal mobile web service. The primary objective is to aid the user in creating a mobile phone mashup application which matches the user's preferences.

\section{Research Backgrounds and Related Works}

While Japanese mobile technologies gain major success in the domestic market, rigid rules for designing mobile web services need to be adhered. Even though in many other countries, internet and mobile technology are converging, the current Japanese mobile technology still has obvious drawbacks and compatibility problems. Various case studies have been observed the i-Mode services outside Japan [1], [2]. The latest 
report shows that European mobile operators have begun to discontinue their i-Mode services. The main reason behind the discontinuation is that i-Mode comes with a fixed business model [3].

Consequently, Japanese mobile users are having no choice but to follow the regulation from carrier-dependent services and profile-specific phones. This research proposes an alternative option to deliver independency to combine internet technology and the ability to create mashup applications from applicable services for mobile phones. A system overview is shown in Fig. 1.

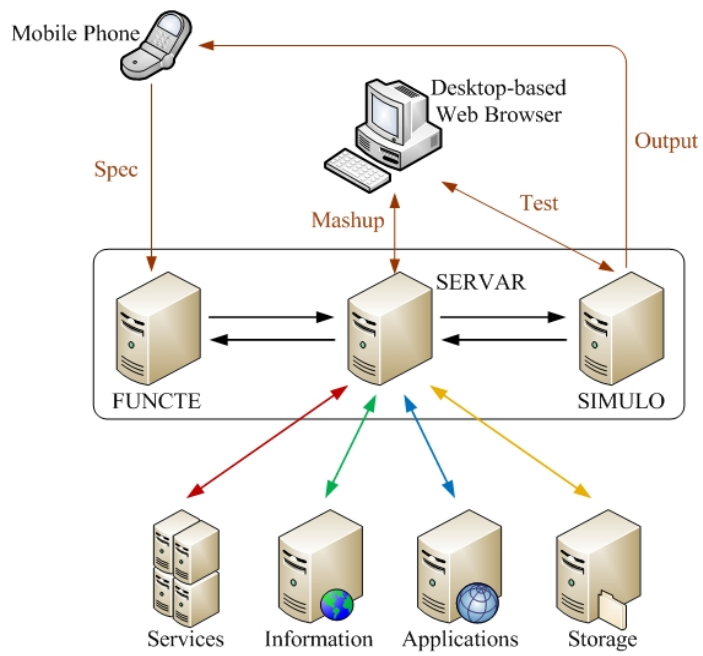

Fig. 1. System overview

1. Our assumption is that users know how to operate their mobile phone browser to a certain level. In some cases, we still require minimal effort from users to run a specification test or make some adjustments which will help the system run correctly on their mobile phone.

2. Specification detection via the user agent string and HTTP headers will be conducted after the mobile phone was registered and accessed the initial page in FUNCTE. The system will automatically detect working functions from this process. Some specifications may need to be tested if they are not available.

3. Users are facilitated by a narrowed choice of mashup modules from the specification detection. All configurations will be passed to the main web mashup engine called SERVAR and match the working services in association with mobile phone's carrier. Using a desktop browser, users can choose applicable services, information and select compatible web application to create their mashup application.

4. After users finish adjusting their mobile service components, simulation of the output page will be display on SIMULO. As soon as the user has completed his/her tuning, the mobile web service page is ready to be published and accessed online.

Although several methods in detecting mobile phone specification have been presented and used worldwide, there is still no central standard for Japanese domestic 
mobile phones. The user agent profile (UAProf) [4] is a set of XML documents that contains information about the agent type and device capabilities. On the other hand, there is an open-source project intended for developers working with the WAP and wireless protocol called WURFL [5]. In fact, it is a manually imported UAProf with additional sources and fixes submitted by other developers. A mobile device web service database called DetectRight [6] provides free and paid subscribers with APIs and analyzer tools which can be integrated with a mobile communication server.

Technology for mobile customization is being developed alongside technology for desktops. The combination of Yahoo pipes and iPhone [7] is suitably leveraging a creation of mobile mashup application via a visual editor. Christian S. Jensen et al [8] demonstrate an idea of user-generated content for mobile services by introducing the STREAMSPIN which enable users to generate their own mobile services from text/photo posting and geospatial information.

\section{Mashup Example and Discussion}

Retrieve Location Information. Similar to the technique applied by Google Map but using the carrier's geo-location web service as a substitute. Some backup service such as DoCoMo Open i-Area, which makes use of radio tower triangulation, may not provide as precise a location as the GPS-based one does. A virtual coordinate rectangle will be used to search for nearby places of interest. Location information are fetched from Wikipedia. The user can also display details of these locations by clicking on the marked dots or switching to text display and links as in Fig. 2.

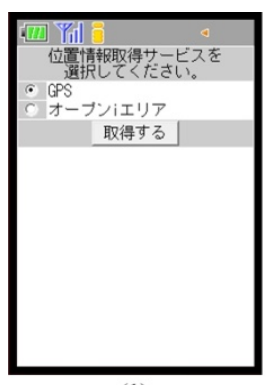

(1)

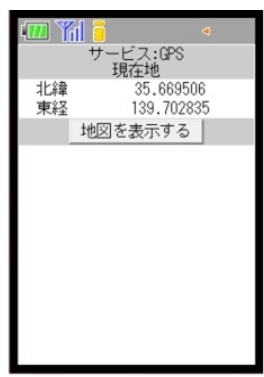

(2)

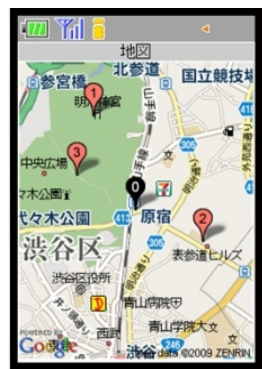

(3)

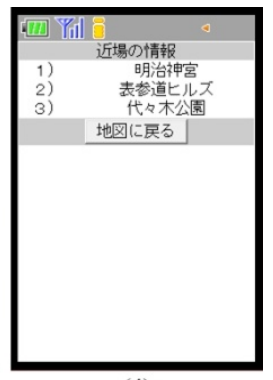

(4)

Fig. 2. Screenshots from mashup example. (1) Select a service from available location-based services (2) Current coordinates (3) Display the map with marked dots (4) Links for details.

Discussion. In our mashup example, there are many untested components which are still in development. We now review some of the limitations of the components used in this evaluation.

1. In our example, we only use HTML. However, some mobile phones can display extended HTML which would allow development of a better user interface.

2. Network latency of each web resource need to be tested and revised. The current method reduces the amount of data received and transmitted. The connection time 
between the mobile phone and the server was very variable. This is because the server has to wait for a response from the requested web service.

3. In providing a map image to the user, the zoom level is not adjustable. This adjustment has to be made manually by the user to fit his/her display area.

4. Search result is limited to a short description. Our intention is not to provide the user with an extra mobile search engine but to have it integrated for other uses.

\section{Conclusion and Future Work}

In order to converge mobility and mashup information, there are several approaches which need to be considered. Most mobile applications are bound with devices' specifications and with the web services they use. Mobile service carriers in Japan tend to keep their services strictly exclusive. Therefore, we developed a web system that addresses device configuration problem at first hand and provides users an option to mash and use their application independently.

Technically, the current system is able to test mobile phone specifications or make specification detection using the user agent string and HTTP headers. Integratible services will be listed during the mashup application creation process. Specifications which have been tested by users are stored on the server and are used as a resource for further development. There are still some components currently under development which include an output simulator, a look and feel configurator and feature adjustability. We are planning to revise the APIs to improve their speed, size and stability in practical use. Using the same ideas, we plan to extend our system to be compatible with more handsets available worldwide.

A mobile phone does not just support text input. It would be interesting to use the microphone and camera on the mobile phone, for example, to support speech recognition or for scanning $2 \mathrm{D}$ barcodes. Better mashup applications could be created for JavaScript-capable phones with accessible low level APIs such as those appeared for the Android or iPhone platforms. Indeed, this study ultimately needs more flexible access to lower level device APIs and higher level web APIs.

\section{References}

1. O'Brien K.J.: Forerunner of mobile Internet, i-mode is fading in Europe, International Herald Tribune (2007),

http://www.iht.com/articles/2007/07/17/business/imode.php

2. Suri, V.R., Sawhney, H.: The internet and its wireless extensions in Japan: the portentous interface between chaos and order. Info. 10(3), 10-21 (2008)

3. Ishii, K.: Internet Use via Mobile Phone in Japan. Telecommunications Policy 28(1), 43-58 (2004)

4. UAProf profile repository, http: //w3development.de/rdf/uaprof_repository/

5. WURFL, http://wurfl. sourceforge.net/

6. DetectRight, http: / /www. detectright.com/

7. Trevor, J.: Doing the Mobile Mash. Computer 41(2), 104-106 (2008)

8. Jensen, C.S., Vincente, C.R., Wind, R.: User-Generated Content: The Case for Mobile Services. Computer 41(12), 116-118 (2008) 